\title{
Successful Treatment of Post-
} Phacoemulsification Descemet's Membrane Detachment Assessed by Anterior Segment Optical Coherence Tomography: A Case Report

\author{
Ayaka Doi $^{a}$ Tadamichi Akagi ${ }^{a, b} \quad$ Akitaka Tsujikawa $^{a}$ \\ aDepartment of Ophthalmology and Visual Sciences, Kyoto University Graduate School of \\ Medicine, Kyoto, Japan; bivision of Ophthalmology and Visual Science, Graduate School of \\ Medical and Dental Sciences, Niigata University, Niigata, Japan
}

\section{Keywords}

Descemet's membrane detachment · Cataract surgery · Gas tamponade · Anterior segment optical coherence tomography

\begin{abstract}
Descemet's membrane detachment (DMD) is a rare but serious complication of phacoemulsification surgery. A small DMD may resolve spontaneously, but extensive DMD often requires intracameral injection of air, nonexpansile gases, or expansile gases. A 92-year-old man who underwent phacoemulsification and aspiration with intraocular lens placement in the right eye had significantly reduced visual acuity, with a hazy cornea after surgery. Anterior segment optical coherence tomography (AS-OCT) examination revealed extensive DMD throughout the cornea. He was treated with intracameral injection of $20 \%$ sulfur hexafluoride. As a result, the Descemet membrane was successfully reattached, and the corneal edema resolved. ASOCT was helpful in confirming the presence and extent of DMD, provided useful information to determine the appropriate treatment, and was useful for monitoring DMD.
\end{abstract}

\section{Introduction}

Descemet's membrane detachment (DMD) is a rare but serious complication of cataract surgery [1]. The incidence of visually significant DMD after phacoemulsification surgery is $0.044 \%$ per year [2]. Descemet's membrane is responsible for maintaining the clarity of the 
cornea; therefore, DMD causes stromal swelling, epithelial bullae, and significant loss of vision. Although there have been some reports of spontaneous reattachment of the Descemet membrane $[3,4]$, the efficacy of pneumodescemetopexy with intracameral air or gas $15-20 \%$ sulfur hexafluoride $\left[\mathrm{SF}_{6}\right]$ or $12-14 \%$ perfluoropropane $\left[\mathrm{C}_{3} \mathrm{~F}_{8}\right]$ ) injection has been reported in severe cases [5]. If the cornea is relatively clear, the presence of DMD can be easily detected by slit-lamp biomicroscopy. However, in the presence of severe corneal edema, anterior segment optical coherence tomography (AS-OCT) is useful for confirming the diagnosis and choosing an appropriate treatment [6].

We describe a patient with extensive DMD following phacoemulsification and aspiration (PEA) with intraocular lens (IOL) placement, which was diagnosed using AS-OCT and managed successfully with intracameral injection of $20 \% \mathrm{SF}_{6}$.

\section{Case Presentation}

A 92-year-old male patient underwent superotemporal clear-corneal PEA with posterior chamber IOL implantation in the right eye. Although partial zonular dehiscence occurred due to a weak Zinn's zonule, the surgery was performed successfully, and the IOL was implanted in the capsular bag. The patient presented with a hazy cornea in the right eye after the surgery and was referred to our hospital on postoperative day 3.

The best-corrected visual acuity was hand motion at $30 \mathrm{~cm}$, and the intraocular pressure in the right eye was $10 \mathrm{~mm} \mathrm{Hg}$. Although diffuse corneal edema was noted and DMD was observed on slit-lamp examination, the details were unclear (shown in Fig. 1a). AS-OCT using Tomey CASIA SS-1000 (Tomey Corporation, Nagoya, Japan) revealed extensive DMD throughout the cornea but no obvious scrolling. Since the DMD was large and visual impairment was severe, we opted for surgical intervention. In the operating room, the anterior chamber was filled with $20 \%$ nonexpansile $\mathrm{SF}_{6}$ through a superonasal paracentesis tract, which was created under AS-OCT guidance in an area where the Descemet membrane was attached, without any complications. The patient was maintained in the supine position after the operation and was administered eye drops of betamethasone, moxifloxacin, bromfenac, atropine, and ripasudil, a Rho-associated protein kinase inhibitor, to heal the endothelial cells. Three days postoperatively, $\mathrm{SF}_{6}$ filled $40 \%$ of the anterior chamber, and AS-OCT showed partial resolution of the DMD (shown in Fig. 1b). One month later, no gas was detected in the anterior chamber, and the DMD had completely disappeared (shown in Fig. 1c). Corneal edema resolved, and the visual acuity improved to 20/20 with a significant improvement in the clearance of the cornea, although the corneal endothelial cell count reduced from 2,307 cells $/ \mathrm{mm}^{2}$ at the examination before PEA + IOL at the previous hospital to 1,264 cells $/ \mathrm{mm}^{2}$.

\section{Discussion}

Small DMDs can resolve spontaneously, but large DMDs can lead to loss of vision and require surgical intervention. Although several surgical options, such as pneumodescemetopexy using air, $\mathrm{SF}_{6}$, or $\mathrm{C}_{3} \mathrm{~F}_{8}$; tamponade with viscoelastic substances or perfluorocarbons; and suturing with 10-0 nylon, have been reported for the treatment of DMD, pneumodescemetopexy with intracameral air or $20 \% \mathrm{SF}_{6}$ is preferred mainly due to its ease of execution and good outcomes [5]. However, it is still controversial whether air or $\mathrm{SF}_{6}$ gas is better for the treatment of DMD. Schaub et al. [7] reported that the use of $20 \% \mathrm{SF}_{6}$ significantly reduced the rate of graft detachment requiring rebubbling after Descemet membrane

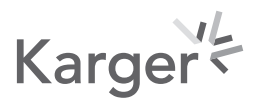




\section{Case Reports in Ophthalmology}

\begin{tabular}{l|l}
\hline Case Rep Ophthalmol 2021;12:611-615 \\
\hline DOI: 10.1159/000517351 & $\begin{array}{l}\text { @ } 2021 \text { The Author(s). Published by S. Karger AG, Basel } \\
\text { www.karger.com/cop }\end{array}$ \\
\hline
\end{tabular}

Doi et al.: Post-Phacoemulsification Descemet's Membrane Detachment Assessed by AS-OCT

a Before SF6 injection
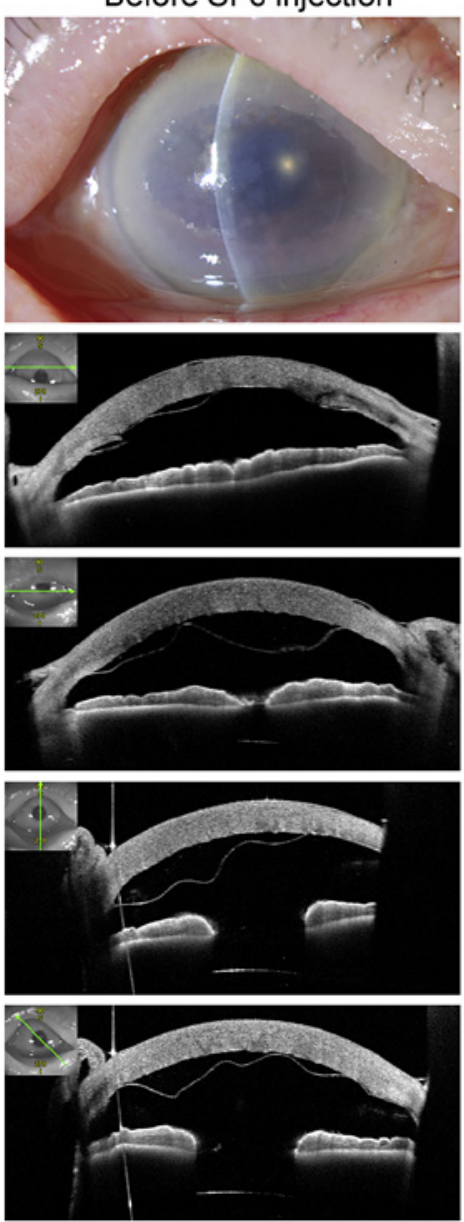

b 3 days after SF6 injection
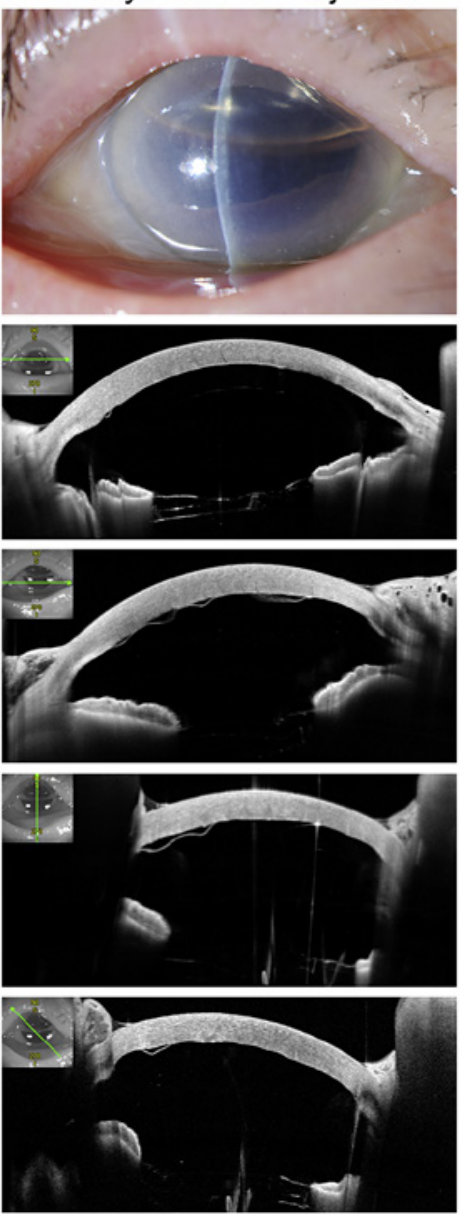

c 1 month after SF6 injection
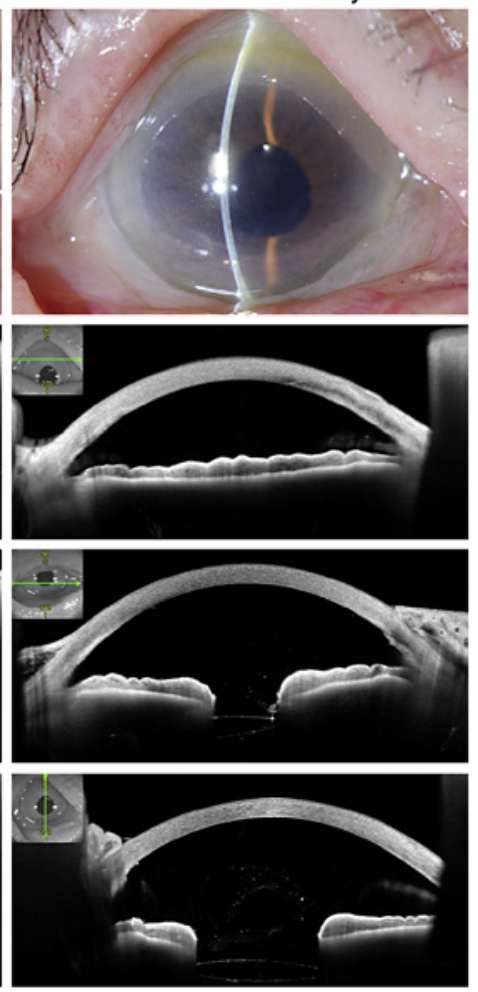

Fig. 1. Clinical course of DMD before and after intracameral $20 \% \mathrm{SF}_{6}$ injection. Slit-lamp photographs (top row) and AS-OCT images (second row and below) before (a), 3 days after (b), and 1 month after SF $_{6}$ injection (c). a Before $\mathrm{SF}_{6}$ injection, severe corneal edema and DMD are observed, and AS-OCT images show extensive DMD and a thickened cornea. b Approximately $40 \%$ of $\mathrm{SF}_{6}$ gas exists in the anterior chamber, and corneal edema persists 3 days after $\mathrm{SF}_{6}$ injection. AS-OCT images show that most of the Descemet membrane has reattached, but partial DMD exists inferiorly. c Corneal edema has improved 1 month after $\mathrm{SF}_{6}$ injection. No DMD is observed in AS-OCT images. The extent of mydriasis is different during the scan periods, but the scan locations are nearly identical and in the same row as seen in the small windows in the upper left corner of the AS-OCT images. DMD, Descemet's membrane detachment; $\mathrm{SF}_{6}$, sulfur hexafluoride; AS-OCT, anterior segment optical coherence tomography.

endothelial keratoplasty (DMEK) compared with the use of air in their retrospective study including 854 eyes that underwent DMEK. $\mathrm{SF}_{6}$ was suspected to be more toxic to corneal endothelial cells and induced more inflammation than air in animal studies [8], whereas other reports showed that there were no significant differences in corneal endothelial cell loss between the $20 \% \mathrm{SF}_{6}$ and air groups in a clinical setting $[7,9]$. More recently, EinanLifshitz et al. [10] reported that in their retrospective study including 82 patients who underwent DMEK, air tamponade had no inferiority in the rebubbling rate, and that air was a better tamponading agent because it was a readily available and short-acting agent. In our patient, a single intracameral injection of $\mathrm{SF}_{6}$ gas was effective in resolving corneal edema rapidly without IOP elevation and the need for rebubbling. However, the reduction in the 
number of endothelial cells was $45 \%$, which was slightly larger than that reported in previous studies on DMEK $(30 \pm 11 \%)$ [9]. Although the main cause of endothelial cell loss in our patient may be the PEA + IOL procedure, we should pay attention to the possible endothelial cell damage during the treatment for DMD.

Extensive DMD induces corneal edema and opacification; consequently, the diagnosis of DMD on slit-lamp examination can be difficult. AS-OCT is a useful tool for confirming the presence and extent of DMD, which can help determine the appropriate treatment [11]. Moutsouris et al. [12] reported that AS-OCT was able to add diagnostic information in 36\% of eyes for which definitive information could not be obtained by slit-lamp biomicroscopy alone. Kumar et al. [13] proposed the AS-OCT-based algorithm, which is based on the height, extent, and length of DMD, with or without pupillary axis involvement, for deciding the treatment. In our patient who had extensive DMD with diffuse corneal edema, AS-OCT was useful for confirming DMD and evaluating its extent. AS-OCT was also useful for identifying the intracameral injection site where the Descemet membrane was attached and for monitoring the recurrence of DMD after treatment.

\section{Conclusion}

AS-OCT was useful for diagnosing DMD and determining treatment, and intracameral injection of $20 \% \mathrm{SF}_{6}$ was efficacious for the treatment of large DMD.

\section{Acknowledgement}

We would like to thank Editage (http://www.editage.com) for editing and reviewing the manuscript for English language.

\section{Statement of Ethics}

The protocol for this retrospective study was approved by the Institutional Review Board and Ethics Committee of the Kyoto University Graduate School of Medicine (approval number R2652). All clinical procedures were conducted according to the principles of the Declaration of Helsinki. Written informed consent was obtained from the patient prior to the procedure, and possible complications were explained. Written informed consent was obtained from the patient for publication of this case report and any accompanying images.

\section{Conflict of Interest Statement}

The authors declare that there is no conflict of interest regarding the publication of this article.

\section{Funding Sources}

This work was supported by the Japan Society for the Promotion of Science (JSPS) KAKENHI, Grant Number 19K09968 (T.A.). The funder had no role in the study design, data collection, analysis, decision to publish, or preparation of the manuscript. 


\section{Author Contributions}

Ayaka Doi contributed to data acquisition, literature search, and manuscript draft. Tadamichi Akagi performed the surgery and was a major contributor in conception and design, interpretation of data, and writing the manuscript. Akitaka Tsujikawa was involved in revising the manuscript. All authors were directly involved in the care of the patient and read and approved the final manuscript.

\section{References}

1 Theodore FH. Corneal complications after cataract surgery. Int Ophthalmol Clin. 1964;4:913-48.

2 Ti SE, Chee SP, Tan DT, Yang YN, Shuang SL. Descemet membrane detachment after phacoemulsification surgery: risk factors and success of air bubble tamponade. Cornea. 2013;32(4):454-9.

3 Ball JL, Stewart 0, Taylor R. Spontaneous reattachment of extensive Descemet's membrane detachment following uneventful phacoemulsification surgery. Eye. 2004;18(9):962.

4 Pan JC, Au Eong KG. Spontaneous resolution of corneal oedema after inadvertent "descemetorhexis" during cataract surgery. Clin Exp Ophthalmol. 2006;34(9):896-7.

5 Chow VW, Agarwal T, Vajpayee RB, Jhanji V. Update on diagnosis and management of Descemet's membrane detachment. Curr Opin Ophthalmol. 2013;24(4):356-61.

6 Singh A, Vanathi M, Sahu S, Devi S. Intraoperative OCT assisted descemetopexy with stromal vent incisions and intracameral gas injection for case of non-resolving Descemet's membrane detachment. BMJ Case Rep. 2017;2017:bcr2016217268.

7 Schaub F, Enders P, Snijders K, Schrittenlocher S, Siebelmann S, Heindl LM, et al. One-year outcome after Descemet membrane endothelial keratoplasty (DMEK) comparing sulfur hexafluoride (SF6) $20 \%$ versus 100\% air for anterior chamber tamponade. Br J Ophthalmol. 2017;101(7):902-8.

8 Landry H, Aminian A, Hoffart L, Nada O, Bensaoula T, Proulx S, et al. Corneal endothelial toxicity of air and SF6. Invest Ophthalmol Vis Sci. 2011;52(5):2279-86.

9 Güell JL, Morral M, Gris O, Elies D, Manero F. Comparison of sulfur hexafluoride 20\% versus air tamponade in Descemet membrane endothelial keratoplasty. Ophthalmology. 2015;122(9):1757-64.

10 Einan-Lifshitz A, Sorkin N, Boutin T, Showail M, Borovik A, Jamshidi F, et al. Comparison of sulfur hexafluoride (SF6) and air tamponade in noniridectomized Descemet membrane endothelial keratoplasty. Cornea. 2018; 37(3):273-6.

11 Sharifipour F, Nassiri S, Idan A. Descemet's membrane detachment management following trabeculectomy. J Ophthalmic Vis Res. 2016;11(3):323-5.

12 Moutsouris K, Dapena I, Ham L, Balachandran C, Oellerich S, Melles GR. Optical coherence tomography, Scheimpflug imaging, and slit-lamp biomicroscopy in the early detection of graft detachment after Descemet membrane endothelial keratoplasty. Cornea. 2011;30(12):1369-75.

13 Kumar DA, Agarwal A, Sivanganam S, Chandrasekar R. Height-, extent-, length-, and pupil-based (HELP) algorithm to manage post-phacoemulsification Descemet membrane detachment. J Cataract Refract Surg. 2015; 41(9):1945-53.

\section{Karger' ${ }^{\prime}$}

